УДК $621.396 .96: 004.94$

\title{
ПОВЫШЕНИЕ ТОЧНОСТИ RFID-ЛОКАЛИЗАЦИИ ПУТЕМ КОМБИНИРОВАНИЯ НЕСКОЛЬКИХ АЛГОРИТМОВ, ОБРАБАТЫВАЮЩИХ ИЗМЕРИТЕЛЬНУЮ ИНФОРМАЦИЮ РАЗЛИЧНЫХ ВИДОВ
}

\author{
Д. А. САВОЧКИН, Ю. Б. ГИМПИЛЕВИЧ \\ Севастопольский национальный технический университет, \\ Украина, Севастополь, 99053, ул. Университетская, 33
}

\begin{abstract}
Аннотация. В статье рассмотрен вопрос двумерной локализации объектов с использованием технологии радиочастотной идентификации (RFID, radio frequency identification). Одной из важных проблем разработки систем RFID-локализации является выбор алгоритма локализации и вида измерительной информации. Обычно разработчики стремятся найти такую комбинацию алгоритма и вида измерительной информации, которая позволяет достичь максимальной точности локализации для конкретного сценария. Однако в некоторых случаях существуют несколько комбинаций алгоритмов и видов измерений с одинаково высокой точностью. Для упрощения проблемы выбора и дополнительного повышения точности был разработан метод комбинированной локализации. Метод основан на усреднении оценок позиций, сформированных несколькими точечными и зонными алгоритмами, обрабатывающими различные измерения. В данной работе использованы три точечных и три зонных алгоритма: алгоритм $k$ ближайших соседей, алгоритм трилатерации, алгоритм пересечений, машина опорных векторов, искусственная нейронная сеть и наивный байесовский классификатор. В качестве входных данных для алгоритмов использованы измерения в виде мощности полученного сигнала, коэффициента считывания и факта наличия ответа от меток. При эксперименте выявлено, что предложенный метод позволяет уменьшить среднюю ошибку на $15 \%$ и максимальную ошибку на $14 \%$ по сравнению с наилучшим одиночным алгоритмом.
\end{abstract}

Ключевые слова: радиочастотная идентификация; RFID; локализация; позиционирование; измерительная информация; комбинированный метод; уровень ответного сигнала

\section{ВВЕДЕНИЕ}

В последнее время системы пространственной двухмерной локализации (позиционирования) в закрытых пространствах становятся все более востребованными в различных сфеpax экономики, особенно в промышленном секторе. Такие системы используются для поиска и отслеживания объектов внутри помещений, например, товаров на складе и в магазине, книг в библиотеке, персонала на работе, пациентов в больнице и т.д. [1].

Задача локализации в закрытых пространствах может быть решена с использованием различных технологий, однако многие из них имеют существенные ограничения. Например системы глобальной спутниковой навигации практически неприменимы внутри помещений, поскольку их точность локализации низка в случае присутствия множества помех, таких как стены и потолки. Ультразвуковые [2] и инфракрасные [3] технологии иногда используются внутри помещений, но они могут обеспечивать высокую точность только для объектов, расположенных в зоне прямой видимости передатчика. Беспроводные сети датчиков [4] и беспроводные локальные сети [5] избавлены 


\section{БИБЛИОГРАФИЧЕСКИЙ СПИСОК}

1. Zhou, J.; Shi, J. RFID localization algorithms and applications - a review. J. Intell. Manuf., vol. 20, no. 6, pp. 695-707, Dec. 2009. DOI: 10.1007/s10845-008-0158-5.

2. Priyantha, N. B.; Chakraborty, A.; Balakrishnan, H. The Cricket location-support system. Proc. of MobiCom, Boston, MA, USA, 2000, pp. 32-43. DOI: 10.1145/345910.345917.

3. Vegni, A. M.; Biagi, M. An indoor localization algorithm in a small-cell LED-based lighting system. Proc. of IPIN, 13-15 Nov. 2012, Sydney, Australia. IEEE, 2012, pp. 1-7, DOI: 10.1109/IPIN.2012.6418887.

4. Suo, H.; Wan, J.; Huang, L.; Zou, C. Issues and challenges of wireless sensor networks localization in emerging applications. Proc. ICCSEE, 23-25 Mar. 2012, Hangzhou, China. IEEE, 2012, vol. 3, pp. 447-451, DOI: 10.1109/ICCSEE.2012.44.

5. Youssef, M.; Agrawala, A. The Horus WLAN location determination system. Proc. MobiSys, Seattle, WA, USA, 2005, pp. 205-218. DOI: 10.1145/1067170. 1067193.

6. Banks, J.; Pachano, M.; Thompson, L.; Hanny, D. "The stage is set," RFID applied. Hoboken, NJ: Wiley, 2007, pp. 3-23.
7. Zhang, D.; Xia, F.; Yang, Z.; Yao, L.; Zhao, W. Localization technologies for indoor human tracking. Proc. FutureTech, 21-23 May 2010, Busan, Korea. IEEE, 2010, pp. 1-6. DOI: 10.1109/FUTURETECH.2010.548 2731.

8. Huang, Y.; Brennan, P. V.; Seeds, A. Active RFID location system based on time-difference measurement using a linear FM chirp tag signal. Proc. PIMRC, 15-18 Sept. 2008, Cannes, France. IEEE, 2008, pp. 1-5, DOI: 10.1109/PIMRC.2008.4699805.

9. Ni, L. M.; Liu, Y.; Lau, Y. C.; Patil, A. P. LANDMARC: indoor location sensing using active RFID. Wireless Networks, vol. 10, no. 6, pp. 701-710, Nov. 2004. DOI: 10.1023/B:WINE.0000044029.06344.dd.

10. Cheng, S. H. An indoor positioning system based on active RFID in conjunction with Bayesian network. Proc. ICMLC, 10-13 Jul. 2011, Guilin, China. IEEE, 2011, pp. 386-390. DOI: 10.1109/ICMLC.2011.6016710.

11. Zhen, Z. N.; Jia, Q.-S.; Song, C.; Guan, X. An indoor localization algorithm for lighting control using RFID. Proc. Energy 2030, 17-18 Nov. 2008, Atlanta, GA, USA. IEEE, 2008, pp. 1-6. DOI: 10.1109/ENERGY. 2008.4781041 .

12. Saha, S.; Chaudhuri, K.; Sanghi, D.; Bhagwat, P. Location determination of a mobile device using IEEE 802.11 b access point signals. Proc. WCNC, 16-20 Mar. 2003, New Orleans, LA, USA. IEEE, 2003, vol. 3, pp. 1987-1992. DOI: $10.1109 /$ WCNC.2003.1200692.

13. Kotsiantis, S. B. Supervised machine learning: a review of classification techniques. Proc. of Conf. on Emerging Artificial Intelligence Applications in Computer Engineering, vol. 160, Amsterdam, Netherlands: IOS Press, 2007, pp. 3-24, URL: http://dl.acm.org/citation.cfm? $\mathrm{id}=1566770.1566773$.

14. Brchan, J. L.; Zhao, L.; Wu, J.; Williams, R. E.; Pérez, L. C. A real-time RFID localization experiment using propagation models. Proc. IEEE RFID, 3-5 Apr. 2012, Orlando, FL, USA. IEEE, 2012, pp. 141-148. DOI: 10.1109/RFID.2012.6193042.

15. Shirehjini, A. A. N.; Yassine, A.; Shirmohammadi, S. An RFID-based position and orientation measurement system for mobile objects in intelligent environments. IEEE Trans. Instrum., Meas., vol. 61, no. 6, pp. 1664-1675, Jun. 2012. DOI: 10.1109/TIM.2011.2181912.

16. Ni, L. M.; Zhang, D.; Souryal, M. R. RFID-based localization and tracking technologies. IEEE Wireless Commun., vol. 18, no. 2, pp. 45-51, Apr. 2011. DOI: 10.1109/MWC.2011.5751295.

17. Subramanian, S. P.; Sommer, J.; Schmitt, S.; Rosenstiel, W. RIL-reliable RFID based indoor localization for pedestrians. Proc. SoftCOM, 25-27 Sept. 2008, Split, Croatia. IEEE, 2008, pp. 218-222, DOI: 10.1109/SOFTCOM.2008.4669483.

18. Laaraiedh, M.; Yu, L.; Avrillon, S.; Uguen, B. Comparison of hybrid localization schemes using RSSI, TOA, and TDOA. Proc. European Wireless, 27-29 Apr. 
2011, Vienna, Austria. IEEE, 2011, pp. 1-5, URL: http://ieeexplore.ieee.org/document/5898074/.

19. Macii, D.; Colombo, A.; Pivato, P.; Fontanelli, D. A data fusion technique for wireless ranging performance improvement. IEEE Trans. Instrum., Meas., vol. 62, no. 1, pp. 27-37, Jan. 2013. DOI: 10.1109/TIM.2012.2209918.

20. Savochkin, D. A. Combinational RFID-based localization using different algorithms and measurements. Proc. MIKON, 16-18 Jul. 2014, Gdansk, Poland. IEEE, 2014, pp. 563-566. DOI: 10.1109/MIKON.2014.689 9958.

21. Liu, H.; Darabi, H.; Banerjee, P.; Liu, J. Survey of wireless indoor positioning techniques and systems. IEEE T SYST MAN CY C, vol. 37, no. 6, pp. 1067-1080, Nov. 2007. DOI: 10.1109/TSMCC.2007.905750.

22. Gimpilevich, Y. B.; Savochkin, D. A. RFID indoor positioning system based on read rate measurement information. Proc. ICATT, 16-20 Sept. 2013, Odessa, Ukraine. IEEE, 2013, pp. 546-548. DOI: 10.1109/ICATT. 2013.6650842.

23. Khedo, K. K.; Sathan, D.; Elaheebocus, R.; Subramanian, R. K.; Rughooputh, S. D. Overlapping zone partitioning localisation technique for RFID. Int. J. of UbiComp, vol. 1, no. 2, pp. 20-32, Apr. 2010. DOI: 10.5121/iju.2010.1202.

24. Caceres, M.; Sottile, F.; Spirito, M. A. WLAN-based real time vehicle locating system. Proc. VTC Spring, 26-29 Apr. 2009, Barcelona, Spain. IEEE, 2009, pp. 1-5, DOI: 10.1109/VETECS.2009.5073916.

25. Mitchell, T. M. "Naive Bayes classifier," Machine Learning. McGraw-Hill SEM, 1997, pp. 177-180.

26. Liu, C.-L.; Hao, H.; Sako, H. Confidence transformation for combining classifiers. Pattern Anal. Applic., vol. 7, no. 1, pp. 2-17, Apr. 2004. DOI: $10.1007 /$ s10044-003-0199-5.

27. Gimpilevich, Yu. B.; Savochkin, D. A. Simulation of measuring data obtained from RFID-tags in systems of spatial localization of objects. Radioelectron. Commun. Syst., vol. 59, no. 7, pp. 301-308, 2016. DOI: $10.3103 / \mathrm{S} 0735272716070037$. 